\title{
Urgences
}

\section{Le quotidien}

\section{Jean-Yves Dupuis}

Numéro 9, 1er trimestre 1984

URI : https://id.erudit.org/iderudit/025133ar

DOI : https://doi.org/10.7202/025133ar

Aller au sommaire du numéro

Éditeur(s)

Urgences

ISSN

0226-9554 (imprimé)

1927-3924 (numérique)

Découvrir la revue

Citer ce document

Dupuis, J.-Y. (1984). Le quotidien. Urgences, (9), 27-34.

https://doi.org/10.7202/025133ar

Ce document est protégé par la loi sur le droit d'auteur. L'utilisation des services d'Érudit (y compris la reproduction) est assujettie à sa politique d'utilisation que vous pouvez consulter en ligne.

https://apropos.erudit.org/fr/usagers/politique-dutilisation/
Cet article est diffusé et préservé par Érudit.

Érudit est un consortium interuniversitaire sans but lucratif composé de l'Université de Montréal, l'Université Laval et l'Université du Québec à Montréal. Il a pour mission la promotion et la valorisation de la recherche. https://www.erudit.org/fr/ 
JEAN-YVES DUPUIS

LE QUOTIDIEN 


\section{LE QUOTIDIEN}

J'ai ajouté: il dit ce qu'il a à dire, il ne fait de concessions à personne. J'aime les gens qui ont des tripes aux ventres.

Elle a dit: ouais...

Elle s'en fichait complètement. Moi j'avais parlé pour parler. Un commentaire que j'avais dit tout haut. J'aurais dû deviner comment ça pouvait être loin de ses préoccupations.

Le jour d'après, elle vint me dire que souvent il allait trop loin, qu'il ne trouvait jamais personne à son goût, et autres sornettes semblables. Je savais qu'elle répétait ce que quelqu'un d'autre lui avait dit, mot pour mot, en copiant toutes les idées, puisqu'elle ne connaissait rien de celui dont elle parlait. Elle disait cela pour se rendre intéressante, voulant dire: moi je me suis faite une opinion personnelle. J'ai laissé faire, qu'estce que cela aurait donné de l'attaquer sur ce point-là?

L'autre jour, sur les tomates, ce fut la même chose. J'avais dit: trop, ce n'est pas bon pour la santé. Elle fait semblant de m'écouter, puis elle dit: oui. Mais je sais bien que l'instant d'après elle n'y pense déjà plus. Qu'est-ce que j'ai à toujours me laisser prendre à vouloir discutailler avec elle? Pourtant, depuis le temps, je devrais la connaître, la dernière fois qu'une idée lui a effleuré le cerveau, cela a dû être à propos de la couleur d'une paire de souliers qui jurait avec une robe neuve. Elle a réglé le tout en achetant d'autres souliers. Une partie de sa vie est employée au magasinage, une autre partie pour se laver. Qu'est-ce qu'elle pourrait faire avec mes tomates?

Le plus souvent, je la laisse parler. Je ne réplique pratiquement jamais à ce qu'elle dit, seulement les rares fois où je m'oublie. Il y a longtemps que j'ai renoncé à l'amener à s'intéresser à quelque chose, je sais bien que c'est inutile s'il n'y a pas un profit en vue à court terme. Dans mes mauvais jours, je me dis que rien n'a d!importance, et qu'une fois mort on se ressemble tous, alors à quoi bon essayer de comprendre quelque chose? Bien sûr je ne lui dis rien de tout ça, d'abord je ne 
crois pas qu'elle comprendrait très bien, et puis elle a peur de la mort, elle est tellement sensible, ça lui donne des cauchemars qu'elle dit. Elle me parle de ses amis, de son travail, et tout, moi je sais bien en la voyant qu'elle ne fait que ruminer sa journée. Elle ne semble pas très heureuse, mais elle ne s'intéresse pas à cette question. Elle est si occupée à faire ce qu'il faut, à arriver au lendemain sans avoir rien oublier. Comment elle trouverait du temps pour réfléchir à ces bêtises-là?

Ah, il y a aussi qu'elle a toujours peur de ce qu'on va dire d'elle. Parfois je lui dis, pour la rassurer: essaie donc de contenter tout le monde. Mais cela ne sert à rien. II ne faut surtout pas qu'on la prenne en défaut de porter une vieille robe démodée, ou qu'on l'aperçoive décoiffée ou sans ses dentiers. Si vous voulez l'avoir de votre côté, faites lui des compliments, après vous pourrez tout lui demander. Au contraire, elle ne peut pas tolérer qu'on ne l'accepte pas, elle s'en mord les lèvres, elle rechigne, elle n'en dormira pas de la nuit. Mais tout ça va avec le fait qu'elle est constamment préoccupée de ce qu'elle paraît, jamais elle ne sort sans avoir longtemps étudier son allure. Pour rire je lui dis: tout ce qu'on peut faire!... Mais elle ne fait pas attention. Elle se fiche de ce que je peux bien penser, moi ça m'arrange un peu, c'est reposant de demeurer avec quelqu'un qui n'est pas toujours à vous juger et qui vous laisse en paix.

Hier-soir, je ne me rappelle plus ce que je faisais, je bouffais peut-être, quand elle est arrivée pleurant presque. Quelqu'un lui avait fait la sale blague de lui lancer un oeuf sur sa robe qu'elle mettait pour la première fois. Elle a dit: ils ont tous trouvé ça drôle. J'ai demandé: qui ça? Elle a répondu: tout le monde, tout le monde dans la rue. Et puis elle s'est mise à pleurer pour de vrai, elle semblait tellement à la merci de tout, si fragile, inoffensive. Tiens, j'ai eu pitié d'elle. Quand elle s'est reprise, elle m'a demandé avec quoi elle ferait partir cette tache-là. Je ne connais rien aux tissus, j'ai dit: je ne sais pas. Puis elle a voulu savoir ce que je pensais de ce bonhomme-là. Mais qu'est-ce que vous voulez que je pense des types qui tirent des oeufs. J'ai dit: bof... ou quelque chose de semblable, en tout cas rien de trascendant. Elle a sans doute pensé que ce 
ne serait pas moi qui pourrait la consoler car elle s'est enfermée dans sa chambre un bon moment. Bien sûr, j'aurais pu l'écouter, ça ne m'aurait même pas coûté un effort, mais je ne savais pas très bien ce qu'il fallait dire. Et ensuite j'ai pensé: elle peut bien pleurer un peu, ce n'est pas la fin du monde ce qui lui arrive.

J'ai fait la vaisselle, j'ai tout nettoyé. Avant que j'aie fini, elle est arrivée dans la cuisine, elle avait ce vieux pantalon avec un trou sur chaque genou. Elle a prit une bière et puis elle s'est assise devant la télévision sans dire un mot. D'habitude, elle ne trouve jamais une minute pour s'asseoir avant dix heures. En la voyant, j'ai su qu'elle noyerait sa peine dans la bière. Un instant j'ai parti pour dire: une simple robe! Mais je me suis demandé s'il n'y avait pas autre chose aussi. Et puis je me suis dit: elle peut bien se saouler. Oui, qu'elle se saoule! Et après? Qu'est-ce que ça peut bien faire?

Je suis venu m'installer près d'elle. Après un instant, quand j'en ai eu assez de ce silence, je lui ai demandé, je lui ai dit quelques mots sans importance, seulement pour parler, elle, elle a haussé les épaules, avec une moue fatiguée, pour dire de ne pas lui parler. Mais l'on se connait assez tous les deux pour que je ne fasse pas attention à ces petits détails. Alors j'ai redemandé:

- Qu'est-ce qu'il y a qui ne vas pas?

- Rien. Qu'est-ce que tu veux qu'il y ait?

Et j'ai dit encore:

- Tu as oublié de te laver.

- Je le ferai plus tard.

- Tu ne devrais pas trop attendre, je me demandais si c'était l'évier ou autre chose...

L'ennui, c'est qu'elle connait toutes mes blagues, faudrait 
que je me renouvelle mais je ne suis pas doué pour ça.

- II doit s'être sûrement passé quelque chose pour que tu aies I'air si abattu.

Elle a dit, je suis sûr d'avoir bien entendu:

- Oui, mais ce n'est pas de tes affaires.

Des fois, il lui arrive de ces sautes d'humeur, mais c'est une bonne fille, tout de suite elle est repentante:

- Je n'ai pas voulu dire ça.

Après un moment, elle a dit, comme ça, sans avertir:

- Ce que je peux être niaiseuse!...

Quand elle commence avec ce disque-là, je choisis la plupart du temps de déguerpir, je la laisse avec ses histoires. J'ai toujours été partisan de ce que l'on garde pour soi ces considérations personnelles. Mais hier, j'étais en train, j'ai choisi de dire:

- Bien non, tu n'es pas si pire que ça.

Elle a répliqué, voulant absolument avoir raison:

- Oui, je suis si pire que ça.

Je n'étais pas pour me battre avec elle, alors je l'ai laissée dire, j'ai fait celui qui était bien prêt à entendre d'autres confidences du même genre, mais elle s'est tue. Elle a commencé à m'intriguer, j'ai voulu en savoir plus, toujours ma sale curiosité, j'ai attendu, mais elle n'a rien dit encore.

- Bon, qu'est-ce qui est arrivé?

- II m'a dit... il m'a dit que je n'étais pas payé pour regarder par la fenêtre. II m'a prise tellement par surprise que j'ai 
sursauté. Puis il m'a demandé quelque chose, à propos de je ne sais plus quoi. J'étais glaçée, je n'ai pas été capable de rien dire. Alors il m'a regardée d'un drôle d'air, puis il est allé s'adresser ailleurs.

Voilà, c'est comme ça, rien de grandiose dans sa vie, toujours de petits détails, de petits faits sans importance qui ont ce pouvoir de la rendre triste ou selon, toute pleine de joie. II y a l'avantage cependant qu'elle oublie tout très vite, le lendemain matin elle a le même air habituel comme s'il ne s'était rien passé. C'est un peu désespérant de la voir jour après jour pareille à la veille, répétant les mêmes gestes, ne s'intéressant à rien, indifférente au monde entier. Mais je me suis habitué à elle, je l'aime bien après tout. Quand j'ai envie de l'estimer un peu plus, je me dis qu'au moins elle ne se mêle pas de philosopher sans avoir le courage d'aller jusqu'au bout. Penser, ça ne l'intéresse pas, elle trouve que c'est du temps perdu. Alors moi je dis avec elle: tu as raison, j'ai pensé avant-hier soir, et tu vois, je suis encore ici.

Je I'ai écoutée un bon moment encore, elle a parlé d'un peu n'importe quoi, mais rien de très réjouissant, elle n'avait pas le coeur à rire, mais plutôt à le déverser dans la bière. J'ai regardé les canettes vides s'accumuler devant elle. Je sais que dans ces moments-là elle cherche quelqu'un pour s'épancher. Mais moi ça ne m'a pas dérangé, je n'avais rien d'autre à faire. Et puis, à la fin, elle m'a communiqué sa mélancolie si bien que I'on est ensuite allé se consoler sur l'oreiller. Cela faisait la quatrième fois que nous faisions l'amour ensemble, cinq si je compte celle où nous sommes rentrés tous les deux trop saouls. En fait, on ne couche ensemble que les rares fois où elle traverse ses petites déprimes. Dans ces moments, elle semble avoir tellement besoin d'affection et d'attention, je me sens comme un bon père de famille qui couche avec une petite fille, pas la sienne bien sûr, disons celle du voisin.

II ne se passe vraiment rien d'extraordinaire dans sa vie. Quand je parle d'elle à des amis, je ne trouve rien à dire. Je ne peux tout de même pas parler de sa manie de la propreté, de ses bas qu'elle lave tous les soirs vingt minutes après être 
rentrée, ou de toutes ses petites habitudes. Seulement je pourrais parler de sa gentillesse, de la façon qu'elle a de vous regarder qui n'est jamais de méfiance ou de lassitude, je pourrais parler de sa patience, oui, et aussi de sa manière de vous écouter qui fait que l'on peut parler sans avoir à toujours surveiller ce que l'on dit. Et puis je crois que je l'aime bien un peu, pas pour le crier sur tous les toits, mais juste un peu, comme ça, sans en avoir l'air. Mais allez donc raconter ça, qui va écouter des histoires semblables? 


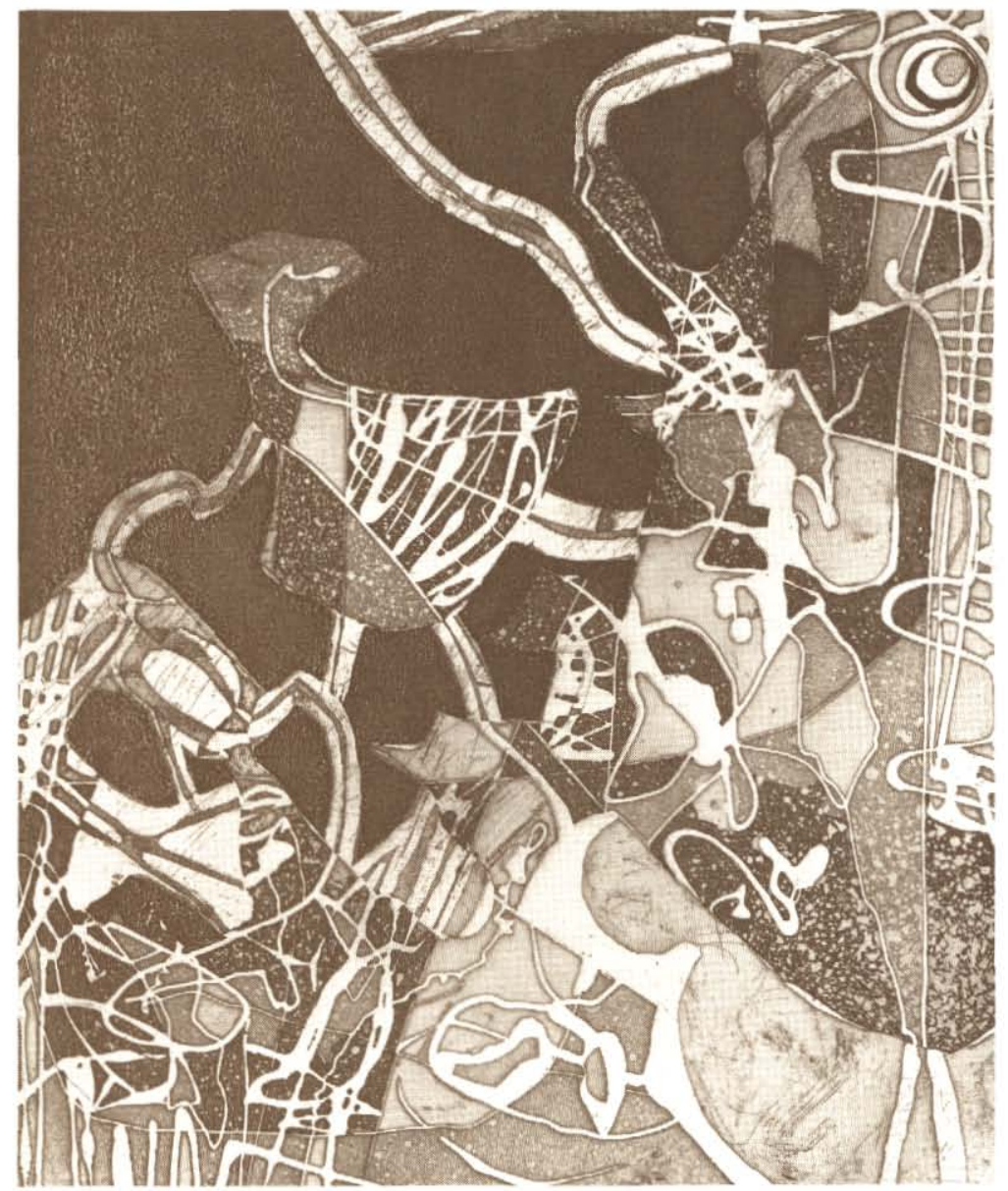

